\title{
CONTRIBUTIONS TO THE BRYOPHYTE FLORA OF CROATIA III. PLITVIČKA JEZERA NATIONAL PARK AND ADJACENT AREAS
}

\author{
A. Alegro ${ }^{1}$, B. PAPP ${ }^{2}$, E. SZURDOKi ${ }^{2}$, V. Š EGOTA ${ }^{3}$, I. Š APIĆ ${ }^{4}$ and \\ J. VUKELIĆ ${ }^{4}$ \\ ${ }^{1}$ Department of Botany and Botanical Garden, Division of Biology, Faculty of Science \\ University of Zagreb, Marulićev trg 20/II, HR-10000 Zagreb, Croatia; antun.alegro@biol.pmf.hr \\ ${ }^{2}$ Department of Botany, Hungarian Natural History Museum \\ H-1476 Budapest, Pf. 222, Hungary; pappbea@bot.nhmus.hu \\ ${ }^{3}$ Institute for Research and Development of Sustainable Ecosystems \\ Jagodno 100a, HR-10410Velika Gorica, Croatia; vsegota@yahoo.com \\ ${ }^{4}$ Department for Forest Ecology and Silviculture, Faculty of Forestry, University of Zagreb \\ Svetošimunska 25, HR-10000 Zagreb,Croatia; jvukelic@sumfak.hr, isapic@sumfak.hr
}

\begin{abstract}
Bryological field surveys in Plitvička jezera National Park and adjacent areas (Slunjčica river and Vrhovinsko polje karst field) carried out in 2012 and 2013 resulted in a list of 207 species (39 liverworts and 168 mosses). Two species are reported for the first time from Croatia (Ephemerum minutissimum and Poblia annotina). Six species are included in the Red data book of European bryophytes (Buxbaumia viridis, Dicranum viride, Hamatocaulis vernicosus, Anomodon rostratus, Rhynchostegiella tenuicaulis and Taxiphyllum densifolium). The first three are listed in the Bern Convention and the EU Habitats and Species Directive as well; in addition, other species, especially Sphagnum spp. rare in SE Europe were also located in the area.
\end{abstract}

Key words: liverworts, mosses, new records for Croatia, threatened species

\section{INTRODUCTION}

This paper represents the continuation of renewed bryological research in Croatia. Although Croatia sometimes considered (e.g. SABOVLJEvić et al. 2001) one of the bryologically best-explored countries in SE Europe, the great majority of data comes from before 1960. On the other hand, comparative statistical analysis of the bryophyte diversity in SE Europe showed that in Croatia a significantly higher number of bryophyte species should be expected (SABOVLJEVIĆ $e t$ al. 2011). Several recent checklists (SABOVLJEVIĆ 2003, 2006, SABOVLJEVIĆ and NATChEVA 2006, SABovlJEviĆ et al. 2008) are mainly new summarisations and compilations of the old data with very sporadic new contributions. This situation aroused an urgent need for gathering new data on Croatia's bryophyte species and their distribution. In order to fill this gap the authors have initiated a series of systematic field studies in different regions of Croatia, and have recently pub- 
lished contributions to the bryophyte flora of the Gorski kotar Region, and of the Northern Velebit Mts (PAPp et al. 2013a, b), as well as list of new taxa for the bryophyte flora of Croatia (PAPP et al. 2013c). Hereby, new contributions for the Lika region, more precisely for the Plitvička jezera National Park and some adjacent areas, Vrhovinsko polje karst field and Slunjčica river, are presented.

Regarding previous bryological surveys of the region concerned, only the tuff barriers of Plitvička jezera had been researched in more detail by Croatian botanist Zlatko Pavletić. The majority of his results were published in coauthority with Croatian zoologist Ivo Matoničkin in a series of papers dealing with the biocenoses and ecology of karst rivers and tuff barriers (MATONIČKIN and PAVLETIĆ 1960, 1961, 1963, 1964, PAvletić 1957). Their species lists for Plitvička jezera are concentrated solely on the tuff barriers, with only 25 bryophyte species (listed in alphabetical order with names used by Pavletić in brackets). These are as follows: Liverworts: Aneura pinguis (L.) Dumort., Conocephalum conicum (L.) Dumort. (Fegatela conica Corda), Jungermannia atrovirens Dumort. (Haplozia riparia (Teyl.) Dum.) and Pellia endiviifolia (Dicks.) Dumort. (P. fabbroniana Raddi); Mosses: Brachythecium rivulare Schimp. (B. rivulare (Bruch.) Br. Eur.), Bryum pseudotriquetrum (Hedw.) P. Gaertn., B. Mey. et Scherb. (B. ventricosum Dicks.), Bryum erythrocarpum complex (B. erythrocarpum Schwägr.), Cinclidotus aquaticus (Hedw.) Bruch et Schimp. (C. aquaticus Br. Eur.), Cratoneuron filicinum (Hedw.) Spruce (C. filicinum Roth), Didymodon tophaceus (Brid.) Lisa (D. tophaceus Jur.), Eucladium verticillatum (With.) Bruch et Schimp. (E. verticillatum Br. Eur.), Fissidens taxifolius Hedw., Fissidens adianthoides Hedw., Fontinalis antipyretica Hedw. (F. antipyretica L.), Gymnostomum aeruginosum Sm. (G. rupestre Schl.), Hygroamblystegium tenax (Hedw.) Jenn. (H. irriguum (Wils.) Loeske), Hymenostylium recurvirostrum (Hedw.) Dixon (Gymnostomum curvirostre (Ehrh.) Lindb.), Leptodictyum riparium (Hedw.) Warnst., Poblia wablenbergii (F. Weber et D. Mohr) A. L. Andrews var. calcarea (Warnst.) E. F. Warb. (Mniobryum calcareum (Warnst.) Limpr.), Orthothecium rufescens (Dicks. ex Brid.) Schimp. (O. rufescens Br. Eur.), Palustriella commutata (Hedw.) Ochyra (Cratoneuron commutatum (Hedw.) Roth), Philonotis calcarea (Bruch et Schimp.) Schimp., Plagiomnium undulatum (Hedw.) T. J. Kop. (Mnium undulatum Weis), Platyhypnidium riparioides (Hedw.) Dixon (P. rusciforme Fleisch.), Rhizomnium punctatum (Hedw.) T. J. Kop. (Mnium punctatum Hedw.).

\section{MATERIAL AND METHODS}

Study area

The area of Plitvička jezera is situated in the mountainous areas of Croatia, on the southern edge of the Mt Mala Kapela. Since 1949 Plitvička jezera are 
protected as national park and in 1979 they were proclaimed a World Natural Heritage by UNESCO. The Park covers 29,685 ha, which includes 200 ha occupied by 16 lakes, 13,320 ha by forests and the rest are grasslands, shrublands and other habitat types. The elevation ranges from $367 \mathrm{~m}$ to $1,279 \mathrm{~m}$ a.s.l. (Seliški vrh peak), with an average altitude of 912 m a.s.l. (BožIČEvIĆ 1994, GušIĆ and MARKOVIĆ 1974, RIĐANOVIĆ 1994). The climate is moderately warm and moist with warm summers. The average annual precipitation is $1,550 \mathrm{~mm}$ with maxima in spring and autumn and the snow cover lasts from November to March. The coldest month is January with an average temperature of $2.2^{\circ} \mathrm{C}$, while the warmest is July (average: $17.4^{\circ} \mathrm{C}$ ) and the annual average is $7.9^{\circ} \mathrm{C}$ (Gušić and MAR Ković 1974, Zaninović 2008). The dominant bedrock is limestone (Triassic, Jurassic and Cretaceous). Different types of beech forests compose the climazonal vegetation, of which beech-fir forests (ass. Omphalodo-Fagetum sylvaticae) are the most characteristic and widespread. The forest stands at Čorkova uvala have never been managed thus considered unique in European context (ANIĆ and MIKAC 2008, Cestar et al. 1983, Plavšić-Goj Ković et al. 1972, PrPIĆ 1972). The area is home to other forest types developed on moist and wet habitats with Alnus glutinosa, Fraxinus excelsior, Salix spp.; on thermophilous habitats with low forests of Fraxinus ornus and Ostrya carpinifolia; on steep slopes with Pinus nigra and P. sylvestris; and on localities with temperature inversion with stands of Picea abies (CESTAR et al. 1976, VUKELIĆ 2012). The grassland vegetation is also very rich, with many associations mainly determined by levels of water availability and $\mathrm{pH}$ (ك̌EGULJA 2005). The lake vegetation is characterised by the dominance of various Chara species, but other macrophytes and helophytes are also present. Very specific for the lakes are the tuff formations, a complex system of barriers and waterfalls, where aquatic bryophyte communities are dominant. One of the best preserved peat bogs in Croatia, Ljeskovačke bare, with a large population of Drosera rotundifolia and other characteristic species (SRDoč et al. 1985, TeŠIĆ et al. 1985), is also situated within the park. The total vascular flora counts 1,267 species with many rare, protected and red-listed species of the Croatian flora (KRGA 1992).

In the study area we also included Vrhovinsko polje, which is situated outside (precisely, alongside the western border) of Plitvička jezera National Park. It is a typical karst field that has its lowest, central part periodically flooded during winter and early spring. It is covered by grassland vegetation of different communities that show a pattern of distribution closely dependent on water amount and soil $\mathrm{pH}$. Considering the diversity of grassland communities and flora, this is one of the richest and most diverse karstlands in Croatia and therefore from 2013 recognised and protected as Natura 2000 site. 
Another locality outside the Park is Slunjčica (or Slušnica) river near the town of Slunj. It is a typical karst river, only $5 \mathrm{~km}$ long, with many tuff barriers and clear unpolluted water. It is a tributary of the Korana river, which is flowing out of the Plitvička jezera lakes. It flows into Korana at Rastoke village, where it forms a complex of cascades and waterfalls. Slunjčica River is protected since 1964 as "significant landscape" as well from 2013 as Natura 2000 site.

\section{Methods}

The collecting trips were made in July 2012 and 2013. The researched area is presented in Figure 1, and particular collecting sites are listed in the following text. During the field trips special attention was paid to collect bryophytes in all habitat types. The specimens are deposited in the Bryophyte Herbarium of the Hungarian Natural History Museum, Budapest (BP) and the Herbarium Croaticum of the University of Zagreb (ZA). The nomenclature follows SCHUMACKER and VÁŇA (2005) for liverworts (except Chiloscyphus pallescens (Ehrh. ex Hoffm.) Dumort., which follows Grolle and Long 2000) and Hill et al. (2006) for mosses. New species for the bryophyte flora of Croatia are given according to general checklists of SE Europe and the Mediterranean (Ros et al. 2007, 2013, Sabovljević 2003, 2006, Sabovljević and Natcheva 2006, SABOVLJEvić et al. 2008) and taking into consideration new records (AlEgRo et al. 2012, Modrić Surina et al. 2012, Papp and Sabovljević 2009, Papp et al. $2013 a, b, c)$.

\section{Collecting sites}

1. Plitvička jezera National Park, Čorkova uvala forest reserve, virgin beech-fir forest (ass. Ompha-

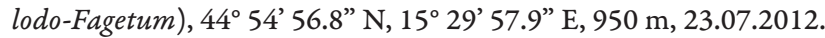

2. Plitvička jezera National Park, Čorkova uvala, Fraxinus excelsior trees at the forest house, $44^{\circ} 54^{\prime}$ $51.3^{\prime \prime} \mathrm{N}, 15^{\circ} 31^{\prime} 43.9$ " E, $885 \mathrm{~m}, 23.07 .2012$.

3. Slunj town, limestone rocks of barrier at the mill on Slunjčica river, $45^{\circ} 06^{\prime} 44.4^{\prime \prime} \mathrm{N}, 15^{\circ} 35^{\prime} 13.7^{\prime \prime}$ E, $240 \mathrm{~m}, 22.07 .2013$.

4. Plitvička jezera National Park, Čorkova uvala, Fraxinus excelsior tree at the forest house, $44^{\circ} 54^{\prime}$ 51.3” N, 15³1' 43.9" E, 885 m, 23.07.2013.

5. Plitvička jezera National Park, Čorkova uvala forest reserve, virgin beech-fir forest (ass. Ompha-

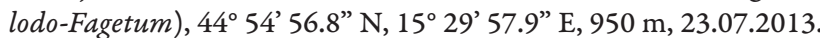

6. Plitvička jezera National Park, Plitvički Ljeskovac, Ljeskovačke bare, transitional peat bog (ass. Drosero-Caricetum echinatae), surrounded by Molinia caerulea meadows, tall herb vegetation of wet habitats (community with Filipendula ulmaria, Lythrum salicaria and Lysimachia vulgaris) and beech-fir forest with limestone rocks, $44^{\circ} 50^{\prime} 57.8^{\prime \prime} \mathrm{N}, 15^{\circ} 36^{\prime} 01.4^{\prime \prime} \mathrm{E}, 640 \mathrm{~m}$, 24.07.2013.

7. Plitvička jezera National Park, Plitvički Ljeskovac, Crna reka rivulet surrounded by stands of Alnus glutinosa and tall herb vegetation dominated by Petasites hybridus, $44^{\circ} 50^{\prime} 31.7^{\prime \prime} \mathrm{N}, 15^{\circ}$ 35 ' 51.9” E, 665 m, 24.07.2013. 
8. Vrhovinsko polje karst field, various grassland and meadow communities, $44^{\circ} 49^{\prime} 04.6^{\prime \prime} \mathrm{N}, 15^{\circ} 29^{\prime}$ 05.8” E, $770 \mathrm{~m}, 25.07 .2013$.

9. Babin potok village, pine forest (ass. Helleboro nigri-Pinetum sylvestrae) on steep slope on dolomite bedrock, $44^{\circ} 50^{\prime} 16.9^{\prime \prime} \mathrm{N}, 15^{\circ} 30^{\prime} 03.1^{\prime \prime} \mathrm{E}, 780 \mathrm{~m}, 25.07 .2013$.

10. Plitvička jezera National Park, Medveđak, beech-fir forest (ass. Omphalodo-Fagetum), $44^{\circ} 53^{\prime}$ 04.7” N, 15³7’ 57.7” E, 640 m, 26.07.2013.

\section{RESULTS AND DISCUSSION}

207 bryophytes ( 39 liverworts and 168 mosses) were collected in Plitvička jezera National Park, along the Slunjčica river near the town of Slunj and in Vrhovinsko polje karst field. 11 recorded taxa were recently published as new for Croatia (PAPp et al. 2013a, b, c) and two are reported here for the first time.

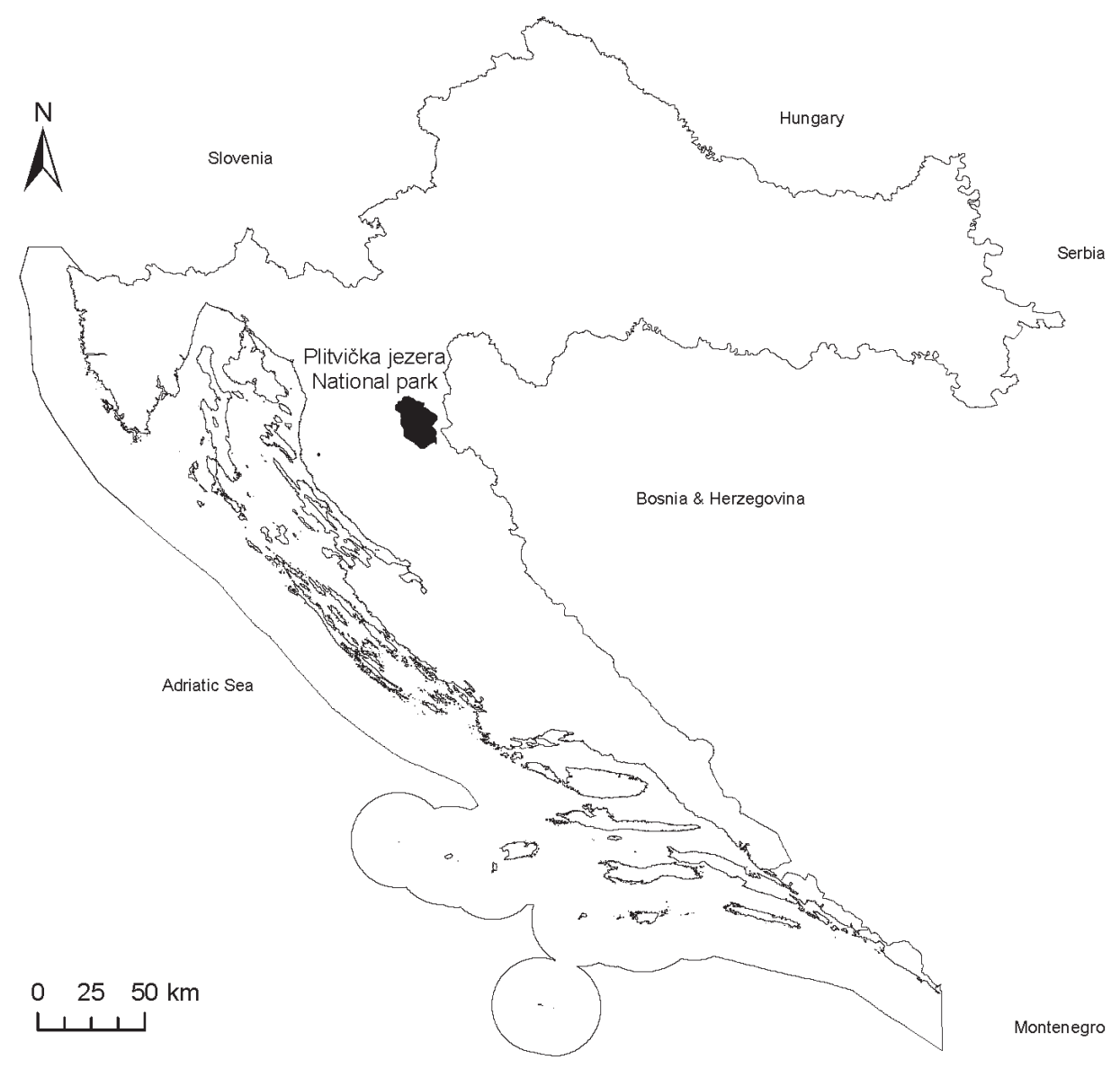

Fig. 1. Location of the study area. 


\section{Hepaticae}

Apometzgeria pubescens (Schrank) Kuwah. - 1, 5: shaded limestone rock Blepharostoma trichophyllum (L.) Dumort. - 5: decaying wood Calypogeia suecica (Arnell et J. Perss.) Müll. Frib. - 1, 5: decaying wood Cephalozia catenulata (Huebener) Lindb. - 1, 5: decaying wood Chiloscyphus coadunatus (Sw.) J. J. Engel et R. M. Schust. - 3, 6: shaded limestone rock Chiloscyphus pallescens (Ehrh. ex Hoffm.) Dumort. - 3: limestone rock at a mill Chiloscyphus profundus (Nees) J. J. Engel et R. M. Schust. - 1, 6, 9: decaying wood Cololejeunea calcarea (Lib.) Schiffn. - 1, 3, 5, 6, 10: shaded limestone rock Cololejeunea rossettiana (C. Massal.) Schiffn. - 1, 5, 10: shaded limestone rock Conocephalum salebrosum Szweyk., Buczkowska et Odrzykoski - 1: limestone rock; 3: limestone rock at a mill; 5: shaded limestone rock

Frullania dilatata (L.) Dumort. - 2: bark of Fraxinus excelsior tree; 6: shaded limestone rock; 10: bark of Fagus sylvatica

Frullania tamarisci (L.) Dumort. - 5: decaying wood; 6: shaded limestone rock

Jungermannia gracillima Sm. - 8: meadow

Jungermannia leiantha Grolle - 1, 5: decaying wood

Jungermannia pumila With. - 3: shaded limestone rock

Lejeunea cavifolia (Ehrh.) Lindb. - 1, 3, 5, 6, 10: shaded limestone rock; 10: bark of Fagus sylvatica Lepidozia reptans (L.) Dumort. - 1, 5: decaying wood

Lophozia badensis (Gottsche) Schiffn. - 6: shaded limestone rock

Lophozia bantriensis (Hook.) Steph. - 1: limestone rock; 6: shaded limestone rock

Lophozia incisa (Schrad.) Dumort. - 1: decaying wood

Lophozia longidens (Lindb.) Macoun - 1: bark of Abies alba

Marchantia polymorpha L. subsp. polymorpha - 7: limestone rock in the stream

Marchantia polymorpha L. subsp. montivagans Bischl. et Boisselier - 7: limestone rock in the stream

Metzgeria conjugata Lindb. - 1, 3, 5, 6, 10: shaded limestone rock; 1: bark of Fagus sylvatica

Metzgeria furcata (L.) Dumort. - 5: bark of Fagus sylvatica; 9: limestone rock; 10: decaying wood Nowellia curvifolia (Dicks.) Mitt. - 1, 5: decaying wood

Pedinophyllum interruptum (Nees) Kaal. - 1, 3, 5, 10: shaded limestone rock

Pellia endiviifolia (Dicks.) Dumort. - 3: limestone rock at a mill; 7: limestone rock in the stream; 8: meadow

Plagiochila asplenioides (L. emend. Taylor) Dumort. - 1, 3: shaded limestone rock

Plagiochila porelloides (Torrey ex Nees) Lindenb. - 1, 3, 5, 6, 10: shaded limestone rock; 1, 10: bark of Fagus sylvatica; 6: on soil

Porella arboris-vitae (With.) Grolle - 1, 10: bark of Fagus sylvatica; 3, 10: shaded limestone rock Porella platyphylla (L.) Pfeiff. - 1: limestone rock; 5: bark of Fagus sylvatica; 10: decaying wood Preissia quadrata (Scop.) Nees - 6: shaded limestone rock

Radula complanata (L.) Dumort. - 1, 10: bark of Fagus sylvatica; 5, 6, 9: shaded limestone rock Riccardia palmata (Hedw.) Carruth. - 1, 5: decaying wood

Scapania aequiloba (Schwägr.) Dumort. - 6: shaded limestone rock

Scapania aspera M. Bernet et Bernet - 3, 5, 6: shaded limestone rock Scapania irrigua (Nees) Nees - 8: meadow

Tritomaria exsecta (Schmidel) Schiffn. ex Loeske -5 : decaying wood 


\section{Musci}

Acaulon muticum (Hedw.) Müll. Hal. - 8: meadow

Amblystegium confervoides (Brid.) Schimp. - 10: shaded limestone rock

Amblystegium serpens (Hedw.) Schimp. - 3: shaded limestone rock and bark of Acer; 7: limestone rock at the stream

Amblystegium subtile (Hedw.) Schimp. - 3: bark of Acer

Anomodon attenuatus (Hedw.) Huebener - 3, 10: shaded limestone rock

Anomodon longifolius (Schleich. ex Brid.) Hartm. - 1, 10: shaded limestone rock

Anomodon rostratus (Hedw.) Schimp. - 1, 5, 10: shaded limestone rock

Anomodon viticulosus (Hedw.) Hook. et Taylor - 1, 3, 5, 9, 10: shaded limestone rock; 3: bark of Acer Antitrichia curtipendula (Hedw.) Brid. - 5, 10: decaying wood

Atrichum undulatum (Hedw.) P. Beauv. - 1, 3: shaded limestone rock; 8: meadow

Aulacomnium palustre (Hedw.) Schwägr. - 6: peat bog; 8: meadow

Barbula crocea (Brid.) F. Weber et D. Mohr - 6: shaded limestone rock

Barbula unguiculata Hedw. - 7: limestone rock at the stream; 8: meadow

Bartramia halleriana Hedw. - 6: shaded limestone rock

Brachytheciastrum velutinum (Hedw.) Ignatov et Huttunen - 1: bark of Fagus sylvatica; 9: on soil Brachythecium mildeanum (Schimp.) Schimp. - 8: meadow

Brachythecium rivulare Schimp. - 7: limestone rock in the stream; 8: meadow

Brachytbecium rutabulum (Hedw.) Schimp. - 1, 5, 10: shaded limestone rock; 1: decaying wood; 3: limestone rock at a mill; 7: limestone rock in the stream; 8: meadow

Brachythecium tommasinii (Sendtn. ex Boulay) Ignatov et Huttunen - 1, 5, 10: shaded limestone rock

Bryoerythrophyllum recurvirostrum (Hedw.) P. C. Chen -7 : limestone rock at the stream

Bryum alpinum Huds. ex With. - 8: meadow

Bryum capillare Hedw. - 1: limestone rock; 6: decaying wood in the meadow; 8: meadow; 9: on soil Bryum elegans Nees - 8: meadow

Bryum moravicum Podp. - 1, 5: shaded limestone rock; 5: decaying wood; 10: bark of Fagus sylvatica

Bryum pseudotriquetrum (Hedw.) P. Gaertn., B. Mey. et Scherb. - 6: peat bog; 7: limestone rock in the stream; 8: meadow

Bryum radiculosum Brid. - 8: meadow

Bryum rubens Mitt. - 8: meadow

Bryum ruderale Crundw. et Nyholm - 8: meadow

Buxbaumia viridis (Moug. ex Lam. et DC.) Brid. ex Moug. et Nestl. - 1, 5: decaying wood

Calliergon giganteum (Schimp.) Kindb. - 6: peat bog; 8: meadow

Calliergonella cuspidata (Hedw.) Loeske - 6, 8: meadow

Campyliadelphus chrysophyllus (Brid.) R. S. Chopra - 6: shaded limestone rock

Campylium stellatum (Hedw.) Lange et C. E. O. Jensen - 6: peat bog; 8: meadow

Campylophyllum calcareum (Crundw. et Nyholm) Hedenäs - 3, 6: shaded limestone rock; 3: bark of Acer

Campylophyllum halleri (Hedw.) M. Fleisch. - 6: shaded limestone rock

Campylopus introflexus (Hedw.) Brid. - 8: margin of meadow

Ceratodon purpureus (Hedw.) Brid. - 8: meadow; 9: on soil

Cinclidotus aquaticus (Hedw.) Bruch et Schimp. - 3: limestone rock at a mill

Cinclidotus fontinaloides (Hedw.) P. Beauv. - 3: limestone rock at a mill

Cinclidotus riparius (Host ex Brid.) Arn. - 3: limestone rock at a mill 
Cirriphyllum crassinervium (Taylor) Loeske et M. Fleisch. - 3, 5, 10: shaded limestone rock Climacium dendroides (Hedw.) F. Weber et D. Mohr - 6: decaying wood in the meadow; 8: meadow Cratoneuron filicinum (Hedw.) Spruce - 3: limestone rock at a mill; 7: limestone rock in the stream; 8: meadow

Ctenidium molluscum (Hedw.) Mitt. - 1, 3, 5, 6, 9, 10: shaded limestone rock

Dicranella staphylina $\mathrm{H}$. Whitehouse - 8: meadow

Dicranella varia (Hedw.) Schimp. - 8: meadow

Dicranodontium denudatum (Brid.) E. Britton - 5: decaying wood

Dicranum bonjeanii De Not. - 6: peat bog; 8: meadow

Dicranum montanum Hedw. - 1, 5: decaying wood

Dicranum scoparium Hedw. -1, 5, 10: shaded limestone rock; 1: bark of Fagus sylvatica; 5: decaying wood; 9: on soil

Dicranum tauricum Sapjegin - 1: decaying wood; 5: bark of Fagus sylvatica

Dicranum viride (Sull. et Lesq.) Lindb. - 10: bark of Fagus sylvatica

Distichium capillaceum (Hedw.) Bruch et Schimp. - 6: shaded limestone rock

Ditrichum flexicaule (Schwägr.) Hampe - 6: shaded limestone rock

Ditrichum gracile (Mitt.) Kuntze - 6: shaded limestone rock

Ditrichum beteromallum (Hedw.) E. Britton - 8: meadow

Drepanocladus aduncus (Hedw.) Warnst. - 8: meadow

Drepanocladus polygamus (Schimp.) Hedenäs - 6: peat bog; 8: meadow

Encalypta streptocarpa Hedw. - 3, 6, 10: shaded limestone rock; 7: limestone rock at the stream

Encalypta vulgaris Hedw. - 6: soil among limestone rocks

Ephemerum minutissimum Lindb. - 8: meadow

Eucladium verticillatum (With.) Bruch et Schimp. - 3: shaded limestone rock

Eurhynchium angustirete (Broth.) T. J. Kop. - 1, 5, 6, 10: shaded limestone rock; 9: on soil

Eurhynchium hians (Hedw.) Sande Lac. - 1, 3, 6, 10: shaded limestone rock; 7: limestone rock at the stream

Eurbynchium striatum (Hedw.) Schimp. - 3: shaded limestone rock

Fissidens adianthoides Hedw. - 6: peat bog; 8: meadow

Fissidens bryoides Hedw. - 8: meadow

Fissidens dubius P. Beauv. - 1, 3, 5, 6, 10: shaded limestone rock

Fissidens taxifolius Hedw. - 5: shaded limestone rock; 8: meadow

Fontinalis antipyretica Hedw. -7 : limestone rock in the stream; 8: meadow

Funaria hygrometrica Hedw. - 8: meadow

Grimmia pulvinata (Hedw.) Sm. - 6: shaded limestone rock

Gymnostomum calcareum Nees et Hornsch. - 3, 6: shaded limestone rock

Gyroweisia tenuis (Hedw.) Schimp. - 3: shaded limestone rock

Hamatocaulis vernicosus (Mitt.) Hedenäs - 6: peat bog

Herzogiella seligeri (Brid.) Z. Iwats. - 1, 5, 9: decaying wood

Homalothecium lutescens (Hedw.) H. Rob. - 8: meadow

Homalothecium philippeanum (Spruce) Schimp. - 5: shaded limestone rock

Homalothecium sericeum (Hedw.) Schimp. - 1, 3, 6, 9: shaded limestone rock; 1: bark of Fagus sylvatica; 5, 10: decaying wood

Homomallium incurvatum (Schrad. ex Brid.) Loeske - 1, 3, 5, 10: shaded limestone rock

Hylocomiastrum pyrenaicum (Spruce) M. Fleisch. - 8: meadow

Hylocomium splendens (Hedw.) Schimp. - 1, 5: shaded limestone rock; 6, 9: on soil

Hypnum cupressiforme Hedw. var. cupressiforme - 1, 9: shaded limestone rock; 5, 6, 10: decaying wood; 8: meadow; 9: on soil; 1, 10: bark of Fagus sylvatica 
Hypnum cupressiforme Hedw. var. lacunosum Brid. - 8: meadow Isopterygiopsis pulchella (Hedw.) Z. Iwats. - 5: shaded limestone rock

Isothecium alopecuroides (Lam. ex Dubois) Isov. - 1, 5, 6, 9: shaded limestone rock; 1, 5, 10: bark of Fagus sylvatica; 10: decaying wood

Isothecium myosuroides Brid. - 1: shaded limestone rock

Leptobryum pyriforme (Hedw.) Wilson - 8: meadow

Leskea polycarpa Hedw. - 3: bark of Acer

Leucodon sciuroides (Hedw.) Schwägr. - 2: bark of Fraxinus excelsior; 6: shaded limestone rock; 10: bark of Fagus sylvatica

Mnium marginatum (Dicks.) P. Beauv. - 1, 3, 10: shaded limestone rock; 7: limestone rock at the stream

Mnium stellare Hedw. - 1, 5: shaded limestone rock; 5: bark of Fagus sylvatica; 7: limestone rock at the stream

Neckera complanata (Hedw.) Huebener - 1, 5, 6: shaded limestone rock; 10: bark of Fagus sylvatica Neckera crispa Hedw. - 1, 3, 6, 10: shaded limestone rock; 1, 5: bark of Fagus sylvatica;10: decaying wood

Neckera pumila Hedw. - 6: shaded limestone rock

Orthothecium rufescens (Dicks. ex Brid.) Schimp. - 6: shaded limestone rock

Orthotrichum affine Schrad. ex Brid. - 2: bark of Fraxinus excelsior

Orthotrichum anomalum Hedw. - 1: shaded limestone rock

Orthotrichum cupulatum Hoffm. ex Brid. - 6: shaded limestone rock

Orthotrichum lyellii Hook. et Taylor - 2: bark of Fraxinus excelsior; 10: bark of Fagus sylvatica

Orthotrichum obtusifolium Brid. - 2: bark of Fraxinus excelsior

Orthotrichum pallens Bruch ex Brid. - 2: bark of Fraxinus excelsior

Orthotrichum pumilum Sw. ex anon. - 2, 4: bark of Fraxinus excelsior

Orthotrichum speciosum Nees - 2: bark of Fraxinus excelsior

Orthotrichum stramineum Hornsch. ex Brid. - 1: bark of Fagus sylvatica

Palustriella commutata (Hedw.) Ochyra - 6: meadow; 7: limestone rock in the stream

Palustriella falcata (Brid.) Hedenäs - 3: limestone rock at a mill

Paraleucobryum longifolium (Hedw.) Loeske -5 : decaying wood

Philonotis fontana (Hedw.) Brid. - 8: meadow

Plagiomnium affine (Blandow ex Funck) T. J. Kop. - 1: shaded limestone rock; 5: decaying wood; 9: on soil

Plagiomnium cuspidatum (Hedw.) T. J. Kop. - 7: limestone rock at the stream; 10: bark of Fagus sylvatica

Plagiomnium ellipticum (Brid.) T. J. Kop. - 6: peat bog

Plagiomnium rostratum (Schrad.) T. J. Kop. - 1, 3: shaded limestone rock

Plagiomnium undulatum (Hedw.) T. J. Kop. - 1, 5, 10: shaded limestone rock

Plagiopus oederianus (Sw.) H. A. Crum et L. E. Anderson - 3, 6: shaded limestone rock

Plagiothecium cavifolium (Brid.) Z. Iwats. - 6: shaded limestone rock

Plagiothecium nemorale (Mitt.) A. Jaeger - 1: shaded limestone rock and bark of Fagus sylvatica

Plagiothecium platyphyllum Mönk. - 5: decaying wood; 10: shaded limestone rock

Plasteurhynchium striatulum (Spruce) M. Fleisch. - 3, 5: shaded limestone rock

Platyhypnidium riparioides (Hedw.) Dixon - 3: limestone rock at a mill; 7: limestone rock in the stream

Pleuridium acuminatum Lindb. - 8: meadow

Pleuridium subulatum (Hedw.) Rabenh. - 8: meadow

Pogonatum urnigerum (Hedw.) P. Beauv. - 8: meadow 
Poblia annotina (Hedw.) Lindb. - 8: meadow Poblia melanodon (Brid.) A. J. Shaw - 8: meadow

Polytrichastrum formosum (Hedw.) G. L. Sm. - 1, 5: decaying wood; 6: on soil; 10: shaded limestone rock

Polytrichum commune Hedw. - 8: meadow

Polytrichum juniperinum Hedw. - 8: meadow

Polytrichum piliferum Hedw. - 8: meadow

Polytrichum strictum Menzies ex Brid. - 6: peat bog

Pseudoscleropodium purum (Hedw.) M. Fleisch. - 6, 9: on soil; 8: meadow

Pterigynandrum filiforme Hedw. - 1: bark of Fagus sylvatica; 5, 10: decaying wood; 5: shaded limestone rock

Pylaisia polyantha (Hedw.) Schimp. - 3: bark of Acer

Racomitrium elongatum Ehrh. ex Frisvoll - 8: meadow

Rhizomnium punctatum (Hedw.) T. J. Kop. - 1, 5: decaying wood

Rhynchostegiella tenuicaulis (Spruce) Kartt. - 1: shaded limestone rock

Rhynchostegium murale (Hedw.) Schimp. - 1, 5, 6, 10: shaded limestone rock

Rhytidiadelphus loreus (Hedw.) Warnst. - 1, 5: shaded limestone rock; 5: decaying wood

Rhytidiadelphus squarrosus (Hedw.) Warnst. - 8: meadow

Rhytidiadelphus triquetrus (Hedw.) Warnst. - 5: shaded limestone rock; 6, 9: on soil

Schistidium crassipilum H. H. Blom - 1, 5, 6, 10: shaded limestone rock

Schistidium elegantulum H. H. Blom - 10: shaded limestone rock

Scorpidium cossonii (Schimp.) Hedenäs - 6: peat bog

Seligeria pusilla (Hedw.) Bruch et Schimp. - 3: shaded limestone rock

Sphagnum capillifolium (Ehrh.) Hedw. - 8: meadow

Sphagnum denticulatum Brid. - 8: meadow

Sphagnum flexuosum Dozy et Molk. - 6: peat bog

Sphagnum palustre L. - 6: peat bog; 8: meadow

Sphagnum squarrosum Crome - 6: peat bog

Sphagnum subnitens Russow et Warnst. - 8: meadow

Sphagnum teres (Schimp.) Ångstr. - 6: peat bog; 8: meadow

Syntrichia ruralis (Hedw.) F. Weber et D. Mohr - 1, 5: shaded limestone rock

Taxiphyllum densifolium (Lindb. ex Broth.) Reimers - 1, 5: shaded limestone rock

Taxiphyllum wissgrillii (Garov.) Wijk et Margad. - 1, 3, 5, 10: shaded limestone rock

Tetraphis pellucida Hedw. - 1, 5: decaying wood

Thamnobryum alopecurum (Hedw.) Gangulee - 1, 3, 5, 10: shaded limestone rock

Thuidium assimile (Mitt.) A. Jaeger - 8: meadow

Tomentypnum nitens (Hedw.) Loeske - 6: peat bog

Tortella bambergeri (Schimp.) Broth. - 5: shaded limestone rock

Tortella inclinata (R. Hedw.) Limpr. - 6, 10: soil among limestone rocks

Tortella tortuosa (Hedw.) Limpr. - 5, 6: shaded limestone rock

Trichodon cylindricus (Hedw.) Schimp. - 8: meadow

Trichostomum brachydontium Bruch - 3: shaded limestone rock

Ulota bruchii Hornsch. ex Brid. - 4: bark of Fraxinus excelsior; 10: bark of Fagus sylvatica

Ulota crispa (Hedw.) Brid. - 10: bark of Fagus sylvatica

Warnstorfia exannulata (Schimp.) Loeske - 8: meadow

Weissia brachycarpa (Nees et Hornsch.) Jur. - 8: meadow

Zygodon rupestris Schimp. ex Lorentz - 5: bark of Fagus sylvatica; 10: decaying wood 
Species reported for the first time from Croatia

During our bryophyte surveys in the period 2011-2012 30 species new for Croatia were found (PAPP et al. 2013a,b,c) 11 of which were recorded again during this research: Conocephalum salebrosum, Lophozia longidens, Dicranodontium denudatum, Plagiothecium platyphyllum, Pterigynandrum filiforme, Racomitrium elongatum, Rhynchostegiella tenuicaulis, Trichodon cylindricus, Tomentypnum nitens, Tortella bambergeri and Zygodon rupestris.

However, the research of the Plitvička jezera lakes and adjacent areas resulted in two additional new species for the Croatian bryophyte flora.

Ephemerum minutissimum is a sub-Atlantic species according to DüLL et al. (1999). In SE Europe it is very rare or probably overlooked, known only from the coastal parts of Montenegro (DüLl et al. 1999, SABOVLJEVIĆ et al. 2008). In the past it was not separated at species level from the related E. serratum, which is known from almost all SE European countries (SABOvLJEvić et al. 2008). From the neighbouring countries E. minutissimum is known from Hungary, where it is treated as near-threatened (NT) in the national red list (PAPP et al. 2010) and from Italy (CORTINI PEDROTTI 1992). Here, the species was found on open soil of meadow edges in Vrhovinsko polje, which is a typical, periodically flooded karstland with a variety of grassland communities determined by water amount and soil $\mathrm{pH}$.

Poblia annotina is a subboreal species (DüLL et al. 1999). It is known from most of the SE European countries (SABOVLJEVIĆ et al. 2008), and from Hungary (PAPp et al. 2010) and Italy (Cortini Pedrotti 1992) as neighbouring countries. This species was also found on soil in Vrhovinsko polje, in its lower, more humid part.

Among the new records for the Croatian bryophyte flora another species, Campylopus introflexus needs to be mentioned. It was found for the first time in Croatia during our field trip in 2013 on decaying Pinus trunk and on soil at the edge of meadow in Vrhovinsko polje, but this record was published separately (ALEGRO et al. 2014). This is an invasive species living on acidic soil, whose expansion started from Britain in 1940 and it keeps spreading towards east (HASSEL and SÖDERSTRÖM 2005). It has already reached northeastern and southeastern Europe according to records from Lithuania (VELLAK et al. 2009), Turkey (YaYıntaş 2009), and Slovenia (SzüCS and Bidló 2014). Currently, no other records are known from the Balkans.

\section{Conservation merits of the bryophyte flora}

Plitvička jezera National Park has a high conservation value due to presence of three species (among in total four in Croatian bryophyte flora) listed in 
the Bern Convention and the European Union Habitats and Species Directive: Buxbaumia viridis, Dicranum viride and Hamatocaulis vernicosus.

Buxbaumia viridis is a boreal, montane species (DÜLL et al. 1999) living on well-decayed wood in humid forests. According to the Red data book of European bryophytes (ECCB 1995) it is a vulnerable species. In spite that historical data in Croatia for this species are scarce (BAUMGARTNER 1938, HoRVAT 1932), recent field surveys showed that this species is not rare in virgin, but even in managed old-growth beech-fir (ass. Omphalodo-Fagetum) and fir (ass. Blechno-Abietetum) forests of the Gorski kotar Region and the Northern Velebit Mts (PAPP et al. $2013 a, b)$, where it has considerably large populations (20-30 sporophytes on 50-100 trees). The species was found in virgin beech-fir forest in Čorkova uvala, where it inhabits dozens of decayed tree trunks.

Dicranum viride is a sub-continental species (DüLL et al. 1999) living as epiphyte on bark of old trees. Historically, it is recorded on the foothills of Mt Papuk (STOIZNER 1870), in the Maksimir park forest in Zagreb (HoRvat 1932) and in Bunjevačka draga in the Velebit Mts (BAUMGartner 1938). On the first two localities it was not confirmed during recent field surveys, while the Bunjevačka draga is a mine suspicious area and therefore inaccessible, but Baumgartner's specimen is preserved in the Hungarian Natural History Museum (BP 31775). Therefore, the Medveđak forest in the Plitvička jezera National Park is currently the single known locality of this species in Croatia. Here it grows in beech-fir forest, in a few small patches on beech bark.

Hamatocaulis vernicosus is a boreal species (DüLL et al. 1999) growing in oligotrophic and mesotrophic, neutral to slightly acidophilous fens and transitional bogs (NEBEL 2001). To date the single finding site of the species in Croatia is Dubravica peat bog in the Hrvatsko zagorje region (NW Croatia) (HoRvaT 1932). It was found there in bog vegetation of ass. Rhynchosporetum albae. Unfortunately, nowadays this bog is only a small remnant of its previous extent and diversity, with uncertain occurrence of $H$. vernicosus. Therefore, the recently found population in Ljeskovačke bare is the only certainly known and really viable population of this species in Croatia. It was found in transition between bog communities Drosero-Caricetum echinatae and Caricetum lasiocarpae. The population is vigorous, with typically developed individuals.

Among other species included in the Red data book of European bryophytes Anomodon rostratus, Rhynchostegiella tenuicaulis and Taxiphyllum densifolium should be mentioned.

Anomodon rostratus is a sub-Mediterranean-montane species (DüLL et al. 1999) occurring on shaded limestone rocks and rock crevices. In the Red data book of European bryophytes it is treated as rare species (ECCB 1995). It was reported for the first time in Croatia (Velebit Mts) by BAUMgARTNER (1938) and 
it was confirmed there during recent surveys (PAPP et al. 2013b). Our new record is the first one outside the Velebit Mts. The species was found in beech-fir virgin forests in Čorkova uvala and Medveđak. The plant grows on strongly shaded limestone rocks in the forest interior, especially in dolina bottoms.

Rhynchostegiella tenuicaulis is a montane species of the temperate zones of Europe (DÜLL 1985). In the Red data book of European bryophytes (ECCB 1995) it is quoted as insufficiently known. The species is rare in SE Europe, known only from Bulgaria, Greece and Romania (SABOVLJEVIĆ et al. 2008). It was recently found in the Velebit Mts on shaded limestone rocks of Štirovača and Jovanovića snižnica ice hole (PAPP et al. 2013b, c). In Plitvička jezera National Park it also grows on shaded limestone rocks, in virgin beech-fir forest in Čorkova uvala.

Taxiphyllum densifolium is an eastern European species distributed sporadically in a range from Slovakia, Poland, Hungary and Romania extending the Caucasus (FREY et al. 2006, VAJDA 1955). Besides Hungary, the only neighbouring country, where it occurs is Serbia (SABOVLJEvić et al. 2008). It was recently reported from Croatia; a specimen of Taxiphyllum wissgrillii, collected near Fiume (Rijeka) in 1909 was revised to T. densifolium (PAPP and SABOVLJEVIĆ 2009). According to the Red data book of European bryophytes (ECCB 1995) it is rare in Europe. We found it in Čorkova uvala in virgin beech-fir forest (ass. Omphalodo-Fagetum) on shaded limestone rocks.

Another group of mosses, which is not included in the Red data book of European bryophytes, but of special local importance, are the peat mosses. All Sphagnum species in Croatia are rare, each with only a few localities or even a single one. Most of them are threatened due to habitat loss and vegetation succession. In the researched area, seven species were found on two localities. The first is Ljeskovačke bare peat bog, where the following species were documented in a transitional bog belonging to ass. Drosero-Caricetum echinatae: Sphagnum flexuosum, S. palustre, S. squarrosum and S. teres. The last one is dominant and this population is the largest one in Croatia. The second locality with peat mosses is Vrhovinsko polje karst field, just outside the border of the national park. Here, the peat mosses grow on the lowest part of the karstland, which is periodically flooded, but completely dry during summer time. The following species were found there: Sphagnum capillifolium, S. denticulatum, S. palustre, S. subnitens and $S$. teres. Both localities are characterised by open habitats, which are threatened by secondary vegetation succession of local trees and shrubs, which will eventually change the water regime, leading to conditions unsuitable for peat mosses.

Calliergon giganteum, Dicranum bonjeanii, Drepanocladus polygamus, Plagiomnium ellipticum, Polytrichum strictum, Scorpidium cossonii and Tomentypnum nitens are additional species growing in wet meadows and bogs, rare in SE Europe, and for this reason of special conservation importance. 
Beyond all the above, in the researched area there are even more bryophyte species rare in SE Europe, which makes the flora peculiar, biogeographically interesting, and highly important for nature conservation. As to flora elements, they are Atlantic and sub-Atlantic species (Calypogeia suecica, Cephalozia catenulata, Barbula crocea, Ephemerum minutissimum, Isothecium myosuroides, Neckera pumila, Plagiothecium platyphyllum, Ulota bruchii and Zygodon rupestris), native to the temperate zones of Europe with montane character (Jungermannia pumila, Tritomaria exsecta, Tortella bambergeri) or they are boreal, subalpine and subarctic species (Lophozia badensis, L. bantriensis, L. longidens, Dicranodontium denudatum, Hylocomiastrum pyrenaicum, Isopterygiopsis pulchella, Orthothecium rufescens, Poblia annotina and Scorpidium cossonii).

\section{CONCLUSIONS}

Plitvička jezera National Park and the adjacent regions possess high bryophyte diversity (with 207 recorded species) making this region comparable with other recently researched areas: Gorski kotar Region with 231 taxa and the Northern Velebit Mts with 191 taxa (PAPP et al. 2013a,b). Here, the high diversity and the occurrence of many rare species are attributed to the habitat diversity. Most widespread are the different forest communities, with beech-fir forests as the zonal ones and represented by several virgin stands. These stands can support vigorous populations of European red-listed bryophytes, including Buxbaumia viridis, Anomodon rostratus, Rhynchostegiella tenuicaulis and Taxiphyllum densifolium. Furthermore, the single known population of Dicranum viride in Croatia also thrives in these forests. Also, important habitat types are the wetlands, with tuff-forming waterfalls, rivulets, lakes, fens, peat bogs, wet and periodically flooded meadows. Each of these habitat types support characteristic bryophyte assemblages with many species rare in SE Europe. In a peat bog Hamatocaulis vernicosus and several Sphagnum species, all rare in Croatia and the Balkans, were found. For the Sphagnum species the periodically flooded meadows at Vrhovinsko polje are of special importance. On open soil near the meadow edge in Vrhovinsko polje Ephemerum minutissimum, a rare (or probably overlooked) species in SE Europe and new for the Croatian bryophyte flora, was found. Poblia annotina is another new species for the country from the same locality.

It needs to be stressed that Plitvička jezera have great conservation value by the occurrence of three bryophyte species listed in the Bern Convention and the European Union Habitats and Species Directive. Furthermore, the presence of many species generally rare in SE Europe, belonging to different flora elements, also marks the importance of this area for bryophyte diversity and its conservation. 


\section{REFERENCES}

Alegro, A., ŠEgota, V. and SAbovljević, M. (2012): Ditrichum gracile (Mitt.) Kuntze. [Croatia]. In: ElLIS, L. T. (ed.): New national and regional bryophyte records, 32. - J. Bryol. 34: 236. http://dx.doi.org/10.1179/1743282012Y.0000000019

Alegro, A., PApp, B., Szurdoki, E., Šegota, V., ŠApić, I. and Vukelić, J. (2014): Campylopus introflexus, a new invasive species in the Croatian flora. - (in press).

ANIĆ, I. and Mi KAC, S. (2008): Struktura, tekstura i pomlađivanje dinarske bukovo-jelove prašume Čorkova uvala. (Structure, texture and regeneration of Dinaric beech-fir virgin forest of Čorkova uvala). - Šumarski list 11-12, 132: 505-515.

Baumgartner, J. (1938): Bryophyten. - In: Degen, A. (ed.): Flora Velebitica, 3, Verlag d. Ungar. Akad. Wiss., Budapest, 595 pp.

BožIČEvić, S. (1994): Hidrogeološki problemi na području Plitvičkih jezera. - In: Proceedings, Plitvička jezera - nacionalno dobro Hrvatske - svjetska baština. Uprava NP Plitvička jezera \& HAZU, Zagreb, pp. 43-51.

Cestar, D., Hren, V., Kovačević, Z., Martinović, J. and Pelcer, Z. (1976): Ekološko-gospodarski tipovi šuma na području Nacionalnog parka Plitvička jezera. - Radovi 28, Šumarski institut, Jastrebarsko, 87 pp.

Cestar, D., Hren, V., Kovačević, Z., Martinović, J., Pelcer, Z., Bezak, K., Krejči, V., Krznar, A., Lindić, V., Medvedović, J. and VrbeK, B. (1983): Prirodni šumski rezervat "Čorkova uvala - Čudinka“. - Radovi 53, Šumarski institut, Jastrebarsko, 44 pp.

Cortini Pedrotti, C. (1992): Check-list of the mosses of Italy. - Flora Medit. 2: 119-221.

DÜLL, R. (1985): Distribution of the European and Macaronesian mosses (Bryophytina) II. Bryol. Beiträge 5: 110-232.

Düll, R., Ganeva, A., Martinčič, A. and Pavletić, Z. (1999): Contributions to the bryoflora of former Yugoslavia and Bulgaria. - Bryol. Beiträge 11: 1-99.

ECCB (1995): Red data book of European bryophytes. - European Committee for Conservation of Bryophytes, Trondheim, $291 \mathrm{pp}$.

Frey, W., Frahm, J.-P., Fischer, E. and Lobin, W. (2006): The liverworts, mosses and ferns of Europe. - Harley Books, Martins, Great Horkesley, Colchester, 512 pp.

Grolle, R. and Long, D. G. (2000): An annotated check-list of the Hepaticae and Anthocerotae of Europe and Macaronesia. - J. Bryol. 22: 103-140.

http://dx.doi.org/10.1179/jbr.2000.22.2.103

Gušıć, B. and MARKović, M. (ed.) (1974): Plitvička jezera - čovjek i priroda. - Nacionalni park Plitvice, Zagreb, $260 \mathrm{pp}$.

HAssel, K. and SöDerströM, L. (2005): The expansion of the alien mosses Orthodontium lineare and Campylopus introflexus in Britain and continental Europe. - J. Hattori Bot. Lab. 97: $183-193$.

Hill, M. O., Bell, N., Bruggeman-Nannenga, M. A., Brugués, M., Cano, M. J., Enroth, J., Flatberg, K. I., Frahm, J.-P., Gallego, M. T., Garilleti, R., Guerra, J., Hedenäs, L., Holyoak, D. T., Hyvönen, J., Ignatov, M. S., Lara, F., Mazimpaka, V., Muñoz, J. and Söderström, L. (2006): An annotated checklist of the mosses of Europe and Macaronesia. - J. Bryol. 28(3): 198-267. http://dx.doi.org/10.1179/174328206X119998

Horvat, I. (1932): Građa za briogeografiju Hrvatske. - Acta Bot. Croat. 7: 73-127.

KRgA, M. (1992): Flora Nacionalnog parka Plitvička jezera. - Plitvički bilten 5: 27-56.

Matoničkin, I. and PAvletić, Z. (1960): Biološke karakteristike sedrenih slapova u našim krškim rijekama. - Geografski glasnik 22: 43-56. 
MatoničKin, I. and Pavletić, Z. (1961): Biljni i životinjski svijet na sedrenim slapovima jugoslavenskih krških voda. - Biološki glasnik 14: 105-128.

MatoničKin, I. and Pavletić, Z. (1963): Prethodna ekološko-biocenološka istraživanja opskrbnih voda plitvičkih jezera. - Acta Bot. Croat. 22: 141-174.

Matoničkin, I. and Pavletić, Z. (1964): Prilozi tipologiji biocenoza na sedrenim slapovima jugoslavenskih krških rijeka. - Acta Musei mac. sc. nat. 9: 122-146.

Modrić Surina, Ž., Randić, M. and Alegro, A. (2012): Calypogeia sphagnicola (Arnell \& J. Pers.) Warnst. \& Loeske. In: Ellis, L. T. (ed.): New national and regional bryophyte records, 31. - J. Bryol. 34: 125. http://dx.doi.org/10.1179/1743282012Y.0000000009

Nebel, M. (2001): Amblystegiaceae. - In Nebel, M. and Phillipi, G. (eds): Die Moose BadenWürttembergs, 2. Verlag E. Ulmer, Stuttgart (Hohenheim), pp. 282-355.

PAPP, B. and SABOVLJEVIĆ, M. (2009): Notes on some new and interesting bryophyte records from Croatia. - J. Bryol. 31: 272-279. http://dx.doi.org/10.1179/037366809X12469790518448

Papp, B., Alegro, A., Šegota, V., Šapić, I. and Vukelić, J. (2013a): Contributions to the bryophyte flora of Croatia I. Gorski kotar Region (W Croatia). - Studia bot. hung. 44: 193-211.

PAPp, B., Alegro, A., ŠEgota, V., ŠAPIĆ, I. and Vukelić, J. (2013b): Contributions to the bryophyte flora of Croatia II. The Northern Velebit. - Studia bot. hung. 44: 213-228.

PApp, B., Alegro, A., ŠEgota, V., ŠApić, I. and Vukelić, J. (2013c): Additions to the bryophyte flora of Croatia. - J. Bryol. 35(2): 140-143. http://dx.doi.org/10.1179/1743282013Y.0000000046

Papp, B., Erzberger, P., Ódor, P., Hoch, Zs., SzÖvéNyi, P., SZUrdoki, E. and Tóth, Z. (2010): Updated checklist and red list of Hungarian bryophytes. - Studia bot. hung. 41: 31-59.

Pavletić, Z. (1957): Ekološki odnosi briofitske vegetacije na slapovima Plitvičkih jezera. - Acta Bot. Croat. 16: 63-88.

Plavšić-Gojković, N., Plavšić, M. and Golubović, U. (1972): Prilog poznavanju biljno-sociološkog sastava i elemenata građe prašumskog rezervata Čorkova Uvala (Nacionalni park Plitvička jezera). - Šumarski List 96(9-10): 348.

PRPIĆ, B. (1972): Neke značajke prašume Čorkova uvala. - Šumarski list 96(9-10): 325-333.

RiĐanović, J. (1994): Geografski smještaj (položaj) i hidrografske značajke Plitvičkih jezera. - In: Proceedings, Plitvička jezera - nacionalno dobro Hrvatske - svjetska baština. Uprava NP Plitvička jezera \& HAZU, Zagreb, pp. 29-42.

Ros, R. M., Mazimpaka, V., Abou-Salama, U., Aleffi, M., Blockeel, T. L., Brugués, M., Cano, M. J., Cros, R. M., Dia, M. G., Dirkse, G. M., El-SaAdawi, W., ErdaĞ, A., Ganeva, A., González-Mancebo, J. M., Herrnstadt, I., Khalil, K., Kürschner, H., Lanfranco, E., Losada-Lima, A., Refai, M. S., Rodríguez-Nuñez, S., Sabovljević, M., Sérgio, C., Shabbara, H., Sim-Sim, M. and Söderström, L. (2007): Hepatics and Anthocerotes of the Mediterranean, an annotated checklist. - Cryptogamie, Bryol. 28(4): 351-437.

Ros, R. M., Mazimpaka, V., Abou-Salama, U., Aleffi, M., Blockeel, T. L., Brugués, M., Cros, R. M., Dia, M. G., Dirkse, G. M., Draper, I., El-SaAdawi, W., Erda Ğ, A., Ganeva, A., Gabriel, R., González-Mancebo, J. M., Granger, C., Herrnstadt, I., Hugonnot, V., Khalil, K., Kürschner, H., Losada-Lima, A., Luís, L., Mifsud, S., Privitera, M., Puglisi, M., Sabovljević, M., Sérgio, C., Shabbara, H. M., Sim-Sim, M., Sotiaux, A., Tacchi, R., Vanderpoorten, A. and Werner, O. (2013): Mosses of the Mediterranean, an annotated checklist. - Cryptogamie, Bryol. 34(2): 99-283.

http://dx.doi.org/doi/10.782/cryb.v34.iss2.2013.99

SABovljević, M. (2003): The hepatic check-list of Croatia. - Arhiv Biol. Nauka 55: 59-66.

Sabovljević, M. (2006): The check list of mosses of Croatia. - Arbiv Biol. Nauka 58: 45-53. 
Sabovljević, M. and Natcheva, R. (2006): A check-list of the liverworts and hornworts of Southeast Europe. - Phytol. Balcan. 12: 169-180.

SAbovljević, M., Ganeva, A., TSAKIri, E. and ŞTefănUȚ, S. (2001): Bryology and bryophyte protection in south-eastern Europe. - Biol. Cons. 101: 73-84. http://dx.doi.org/10.1016/S0006-3207(01)00043-X

Sabovljević, M., Alegro, A., Sabovljević, A., Marka, J. and Vujičić, M. (2011): An insight into diversity of the Balkan peninsula bryophyte flora in the European background. - Rev. Écol. (Terre Vie) 66: 399-413.

Sabovljević, M., Natcheva, R., Tsakiri, E., Dihoru, G., Dragićević, S., ErdaĞ, A. and PAPP, B. (2008): Check-list of the mosses of SE Europe. - Phytol. Balcan. 14: 207-244.

SCHUMACKeR, R. and VÁŇA, J. (2005): The identification keys of the liverworts and hornworts of Europe and Macaronesia. - Sorus, Poznań, 209 pp.

ŠEGULJA, N. (2005) Vegetacija travnjaka, cretišta i močvarnih staništa Nacionalnog parka Plitvička jezera. (Vegetation of grassland, moor and swamp habitats in Plitvička jezera National Park). - Natura Croatica 14 (Suppl. 2): 1-194.

Srdoč, D., Obelić, B., Horvatinčić, N., Culiberg, M., Šercelj, A. and Sliepčević, A. (1985): Radiocarbon dating and pollen analyses of two peat bogs in the Plitvice national park. - Acta Bot. Croat. 44: 41-46.

Stoizner, C. (1870): Nachtrag zu Flora von Slavonien II. - Verh. zool.-bot. Ges. Wien 10: 1009-1013.

SzÜCs, P. and Bidló, A. (2014): Campylopus introflexus (Hedw.) Brid. [Slovenia]. In: ElLIs, L. T. (ed.): New national and regional bryophyte records, 41. - J. Bryol. 36(4): 308. http://dx.doi.org/10.1179/1743282014Y.0000000123

Tešić, Ž., Bogdanović, M., Todorović, M., Stojanović, S. and Gigov, A. (1985): Plitvička tresava i osobine njenog treseta. (The Plitvice peat-bog and the properties of its peat). Zemljište i biljka 34: 159-179.

VAJdA, L. (1955): Plagiothecium densifolium (Lindb.) Broth. in Ungarn. - Mitt. d. Thüring. Bot. Gesell. 1(2-3): 225-230.

Vellak, K., IngerpuU, N., Kannukene, L. and Leis, M. (2009): New Estonian records: liverworts and mosses. - Folia Cryptog. Estonica 45: 91-93.

Vukelić, J. (2012): Šumska vegetacija Hrvatske. - Šumarski Fakultet, Sveučilište u Zagrebu \& Državni zavod za zaštitu prirode, Zagreb, 403 pp.

Yayintaş, Ö. T. (2009): Campylopus introflexus (Hedw.) Brid. [Turkey]. In: Blockeel, T. L. (ed.): New national and regional bryophyte records, 20. - J. Bryol. 31: 55. http://dx.doi.org/10.1179/jbr.2009.31.1.64

ZAninović, K. (ed.) (2008): Klimatski atlas Hrvatske. (Climate atlas of Croatia). - DHMZ, Zagreb, $200 \mathrm{pp}$. 